\title{
Indirekte Bestimmung kurzer Relaxationszeiten von paramagnetischen Ionen in wäßriger Lösung
}

\author{
K. Glemser, U. Haeberlen und R. Hausser \\ I. Physikalisches Institut der Universität Stuttgart \\ (Z. Naturforsch. 24 a, 424-427 [1969]; eingegangen am 16. November 1968)
}

\begin{abstract}
The width of the first Hfs-line of the $\mathrm{Mn}^{++}$EPR-spectrum in aqueous solutions with added diamagnetic and paramagnetic iron group or rare earth ions was measured at $3 \mathrm{~cm}$ wavelength and at $300^{\circ} \mathrm{K}$. The difference $\Delta H_{\mathrm{D}}$ in linewidth of $\mathrm{Mn}^{++}$in the paramagnetic and diamagnetic salt solutions is ascribed to dipolar interaction between $\mathrm{Mn}^{++}$and the other paramagnetic ions e. g. $\mathrm{Co}^{++}$, $\mathrm{Ni}^{++}$and $\mathrm{Ce}^{3+}$. The relaxation times $\tau_{\mathrm{s}}$ of these ions are too short for the EPR-spectrum to be detected in liquids. From the excess linewidth $\Delta H_{\mathrm{D}}$ relaxation times $\tau_{\mathrm{s}}$ for $\mathrm{Co}^{++}, \mathrm{Ni}^{++}$and $\mathrm{Ce}^{3+}$ are estimated which agree well with $\tau_{\mathrm{s}}$ results from proton relaxation measurements in paramagnetic salt solutions.
\end{abstract}

Die Relaxationszeiten $\tau_{\mathrm{s}}$ der Elektronenspins vieler paramagnetischer Ionen sind in wäßrigen Lösungen so kurz, daß kein EPR-Spektrum nachgewiesen werden kann ${ }^{1}$. Eine direkte Messung von $\tau_{\mathrm{s}}$ ist daher nicht möglich. Einige Abschätzungen solch kurzer Relaxationszeiten sind aus Untersuchungen der paramagnetischen Resonanz und Relaxation von Protonen in Lösungen der Ionen der Eisengruppe und der Gruppe der seltenen Erden bekannt ${ }^{2-}$. Um über $\tau_{\mathrm{s}}$ einiger solcher Systeme $-\mathrm{Co}^{++}, \mathrm{Ni}^{++}$ und $\mathrm{Ce}^{3+}$ - weitere Informationen zu erhalten, wird in dieser Arbeit deren Wechselwirkung mit einem zweiten Elektronenspinsystem, dessen EPR-Spektrum in flüssigen Lösungen beobachtet werden kann, untersucht. Für dieses zweite Spinsystem wurde eine wäßrige $\mathrm{MnSO}_{4}$-Lösung gewählt. $\mathrm{Mn}^{++}$besitzt in verdünnten Lösungen ein leicht nachweisbares, voll aufgelöstes Hyperfeinstruktur-(Hfs) Spektrum.

Zur indirekten Bestimmung der Relaxationszeiten von $\mathrm{Co}^{++}, \mathrm{Ni}^{++}$und $\mathrm{Ce}^{3+}$ wurde die Breite $\Delta H$ der ersten Hfs-Linie des $\mathrm{Mn}^{++}$-EPR-Spektrums in $\mathrm{Ab}$ hängigkeit von der Konzentration der Salze $\mathrm{CoSO}_{4}$, $\mathrm{NiSO}_{4}$ und $\mathrm{Ce}\left(\mathrm{NO}_{3}\right)_{3}$ gemessen. Um die Wechselwirkung zwischen $\mathrm{Mn}^{++}$und $\mathrm{Co}^{++}, \mathrm{Ni}^{++}$bzw. $\mathrm{Ce}^{3+}$ (Spin-Spin-Wechselwirkung) von der Wechselwirkung der $\mathrm{Mn}^{++}$-Ionen mit den fluktuierenden elektrischen und magnetischen Feldern der Anionen und $\mathrm{H}_{2} \mathrm{O}$-Dipole der Solvathülle (Spin-Gitter-Wechsel-

1 S. A. Altschuler u. B. M. Kosyrew, Paramagnetische Elektronenresonanz, Harri Deutsch Verlag, Zürich und Frankfurt/M. 1964.

2 L. O. Morgan u. A. W. Nolle, J. Chem. Phys. 31, 365 [1959].

3 N. Bloembergen u. L. O. Morgen, J. Chem. Phys. 34, 842 [1961]. wirkung) zu separieren, wurde außerdem die Breite $\Delta H_{\mathrm{G}}$ der Hfs-Linie in Abhängigkeit von der Konzentration der diamagnetischen Salze $\mathrm{ZnSO}_{4}$ und $\mathrm{La}\left(\mathrm{NO}_{3}\right)_{3}$ gemessen. Unter der Annahme, daß die Spin-Gitter-Relaxation der $\mathrm{Mn}^{++}$-Ionen bei konstanter Temperatur nur von Konzentration und Art der Anionen der gelösten Salze und von der Ionenstärke der Lösung abhängt, kann die Differenz $\Delta H_{\mathrm{D}} \equiv \Delta H-\Delta H_{\mathrm{G}}$ auf die Wechselwirkung zwischen $\mathrm{Mn}^{++}$und $\mathrm{Co}^{++}, \mathrm{Ni}^{++}$bzw. $\mathrm{Ce}^{3+}$ zurückgeführt werden. Die Ionen der dia- und paramagnetischen Salze, die miteinander verglichen werden, sind jeweils von derselben Gruppe der Übergangselemente, sie haben die gleiche Wertigkeit und sind mit derselben Anionenart verbunden.

\section{Durchführung der Experimente und Meßergebnisse}

Die $\mathrm{Mn}^{++}$-Spektren wurden mit einem X-Band EPR-Spektrometer mit $470 \mathrm{~Hz}$ Magnetfeldmodulation aufgenommen. Um den Effekt der Überlagerung der $6 \mathrm{Hfs}$-Linien des $\mathrm{Mn}^{++}$-Spektrums bei zunehmender Linienverbreiterung möglichst klein zu halten, wurde die Breite der ersten Hfs-Linie auf der Seite kleiner Magnetfeldstärke $\left(m_{\mathrm{K}}=+5 / 2\right)$ gemessen. Als Linienbreite $\Delta H$ wird der volle $\mathrm{Ab}$ stand der Extrema der differenzierten Absorptions-

\footnotetext{
${ }^{4}$ Z. Luz u. S. Merвоom, J. Chem. Phys. 40, 1058, 1066 [1964].

5 U. Lindner, Ann. Physik, Leipzig 16, 319 [1965].

${ }^{6}$ G. Held, Diplomarbeit, Universität Stuttgart 1966.

7 F. NOACK, Dissertation, Universität Stuttgart 1967.
} 

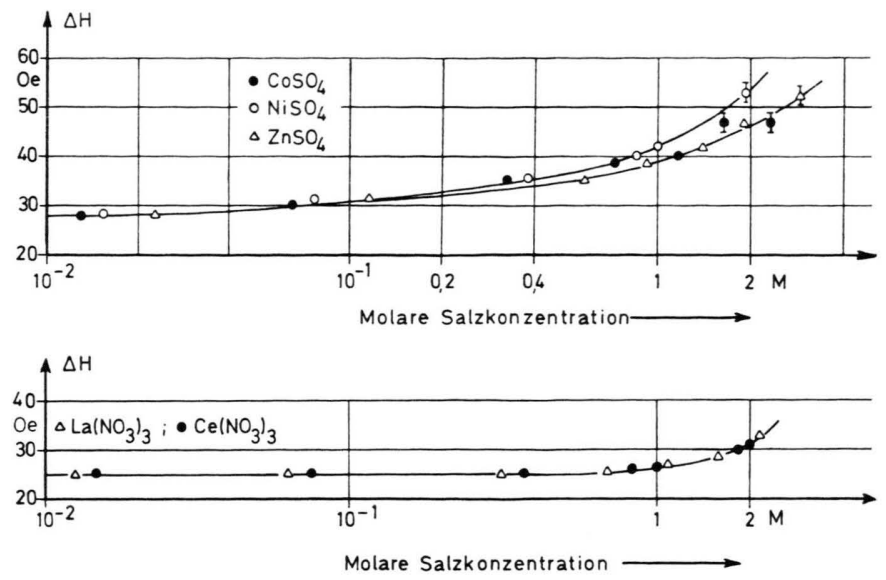

Abb. 1. Breite $\Delta H$ der ersten Hfs-Linie $(m=+5 / 2)$ des $\mathrm{Mn}^{++}$-EPR-Spektrums in einer $3 \cdot 10^{-3}$-mol. $\mathrm{MnSO}_{4}$-Lösung in Abhängigkeit von der Konzentration der Salze $\mathrm{CoSO}_{4}, \mathrm{NiSO}_{4}$ und $\mathrm{ZnSO}_{4}$.

Abb. 2. Breite $\Delta H$ der ersten Hfs-Linie des $\mathrm{Mn}^{++}$. EPR-Spektrums in einer $3 \cdot 10^{-3}$-mol. $\mathrm{MnSO}_{4}$-Lösung in Abhängigkeit von der Konzentration der Salze $\mathrm{La}\left(\mathrm{NO}_{3}\right)_{3}$ und $\mathrm{Ce}\left(\mathrm{NO}_{3}\right)_{3}$. linie definiert. Die Magnetfeldstärke wurde mit einer Hall-Sonde gemessen, diese war mittels Protonenresonanz geeicht. Die Konzentration der $\mathrm{Mn}^{++}$-Ionen wurde konstant gehalten und $\mathrm{zu} 3 \cdot 10^{-3} \mathrm{Mol} / \mathrm{Liter}$ gewählt. Die Untersuchungen erfolgten bei (27 $\pm 1){ }^{\circ} \mathrm{C}$.

Abb. 1 zeigt für je eine Meßreihe die Abhängigkeit der Breite der ersten $\mathrm{Mn}^{++}$-Hfs-Linie von der Konzentration der Salze $\mathrm{CoSO}_{4}, \mathrm{NiSO}_{4}$ und $\mathrm{ZnSO}_{4}$, Abb. 2 entsprechend die Abhängigkeit von der $\mathrm{Ce}\left(\mathrm{NO}_{3}\right)_{3}{ }^{-}$und $\mathrm{La}\left(\mathrm{NO}_{3}\right)_{3}$-Konzentration. Die Linienbreite ist in den $\mathrm{Ce}\left(\mathrm{NO}_{3}\right)_{3}$ - und $\mathrm{La}\left(\mathrm{NO}_{3}\right)_{3}$ - $\mathrm{Lö}$ sungen gleich, d.h. die Wechselwirkung zwischen $\mathrm{Mn}^{++}$und $\mathrm{Ce}^{3+}$ hat keinen meßbaren Einfluß auf die Manganlinie. Die Zunahme der Linienbreite mit wachsender $\mathrm{NO}_{3}$-Konzentration (Abb. 2) kann mit dem Eindringen der Anionen in den $\mathrm{Mn}^{++}$-Hydratkomplex erklärt werden ${ }^{8,9}$. Die elektrischen Felder in der Umgebung der $\mathrm{Mn}^{++}$-Ionen ändern sich dabei stark und führen über die Spin-Bahn- und SpinSpin-Kopplung der 3d-Elektronen zu verstärkter Spin-Gitter-Relaxation.

Analog kann die Hauptzunahme der Linienbreite in Abb. 1 mit der Wechselwirkung zwischen $\mathrm{Mn}^{++}$. und $\mathrm{SO}_{4}^{--}$-Ionen erklärt werden ${ }^{8}$; die Meßpunkte der $\mathrm{NiSO}_{4}$-Lösungen liegen jedoch über denen von $\mathrm{CoSO}_{4}$ und $\mathrm{ZnSO}_{4}$. In Abb. $3 \mathrm{a}-\mathrm{c}$ ist für alle Meßreihen in doppelt logarithmischem Maßstab die Än-

8 R. G. Hayes u. R. J. Myers, J. Chem. Phys. 40, 377 [1964].

9 P. G. Tischkov u. G. P. Wischnewskaya, Soviet Phys.JETP 11, 243 [1960].

Abb. $3 \mathrm{a}-$ c. Änderung $\Delta H-\Delta H_{0}\left(\Delta H_{0}=28\right.$ Oe $)$ der Breite der ersten Hfs-Linie von $\mathrm{Mn}^{++}$in Abhängigkeit von der molaren Konzentration der Salze $\mathrm{CoSO}_{4}, \mathrm{NiSO}_{4}$ und $\mathrm{ZnSO}_{4}$.
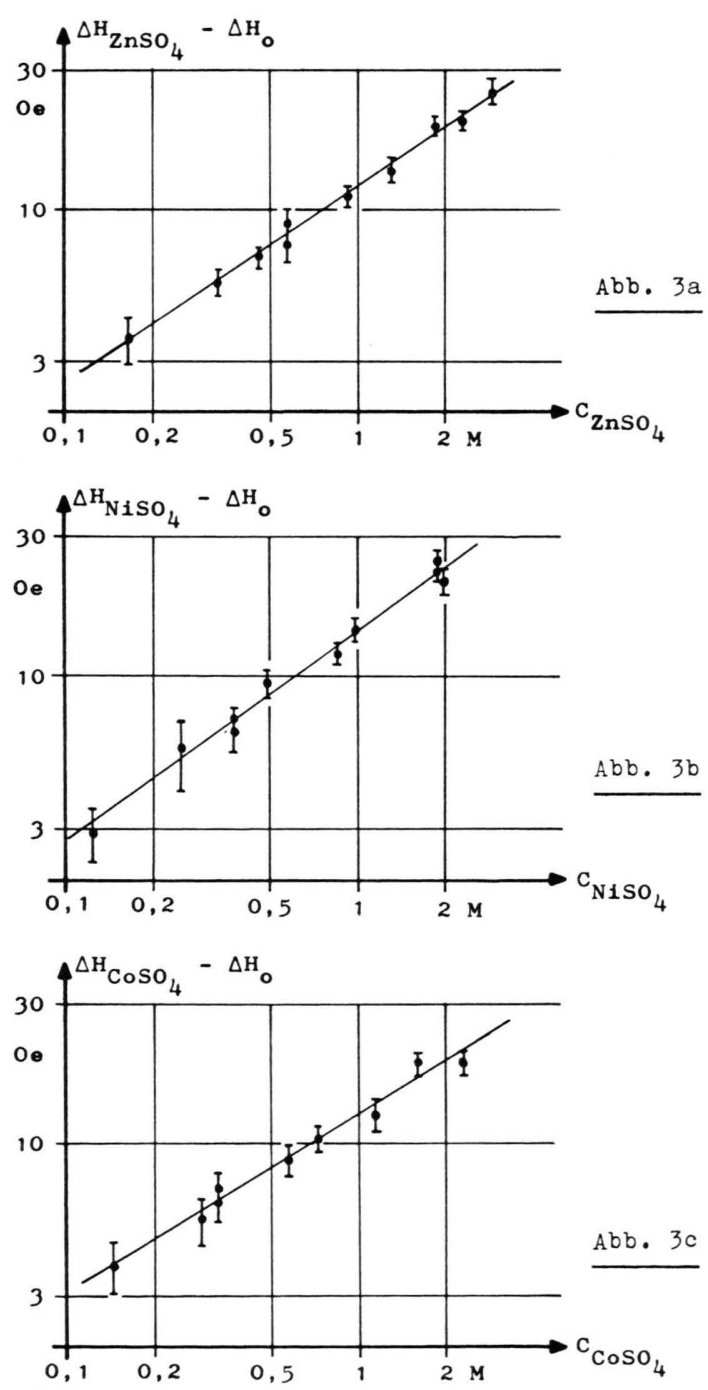
derung $\Delta H-\triangle H_{0}$ gegen die Konzentration der Salze $\mathrm{ZnSO}_{4}, \mathrm{NiSO}_{4}$ und $\mathrm{CoSO}_{4}$ aufgetragen. Die Meßpunkte können jeweils durch eine Gerade verbunden werden; für die weitere Auswertung werden diese Geraden benutzt.

Die Geraden von $\mathrm{NiSO}_{4}\left(\mathrm{Abb} .3 \mathrm{~b}\right.$ ) und $\mathrm{ZnSO}_{4}$ (Abb. 3 a) haben verschiedene Steigung. Der Unterschied

$$
\Delta H_{\mathrm{D}} \equiv\left(\Delta H_{\mathrm{NiSO}_{4}}-\Delta H_{0}\right)-\left(\Delta H_{\mathrm{ZnSO}_{4}}-\Delta H_{0}\right)
$$

ist im Rahmen der Meßgenauigkeit der Konzentration $C_{\mathrm{S}}$ der gelösten Salze proportional:

$$
\Delta H_{\mathrm{D}}=B \cdot C_{\mathrm{S}} \text {, wobei } B=3,7 \cdot 10^{-21} \frac{\mathrm{Oe}}{\mathrm{Ionen} / \mathrm{cm}^{3}} \text { ist. }
$$

Den Beitrag $\Delta H_{\mathrm{D}}$ zur Breite der $\mathrm{Mn}^{++}$-Hfs-Linie schreiben wir der Wechselwirkung zwischen $\mathrm{Mn}^{++}$ und $\mathrm{Ni}^{++}$zu. Mit dem Ergebnis des nächsten $\mathrm{Ab}$ schnitts kann aus $\Delta H_{\mathrm{D}}$ eine mittlere Elektronenspinrelaxationszeit $\tau_{\mathrm{s}}$ der $\mathrm{Ni}^{++}$-Ionen berechnet werden.

Der Unterschied zwischen den Geraden von $\mathrm{CoSO}_{4}$ (Abb. $3 \mathrm{c}$ ) und $\mathrm{ZnSO}_{4}$ (Abb. 3 a) zeigt keinen konzentrationsabhängigen Verlauf, die Differenzen streuen im Bereich des Meßfehlers von ca. 1 Oe. Eine Wechselwirkung zwischen $\mathrm{Mn}^{++}$und $\mathrm{Co}^{++}$kann daher auf diesem Wege nicht nachgewiesen werden.

\section{Theorie}

Die Rechnung, welche den Zusammenhang zwischen $\Delta H_{\mathrm{D}}$ und $\tau_{\mathrm{s}}$ herstellt, kann in Analogie zur Berechnung der Relaxationszeiten von Protonen in Lösungen paramagnetischer Ionen durchgeführt werden. Die Brownsche Bewegung der Ionen wird dabei durch eine isotrope statistische Translation beschrieben ${ }^{10,11}$.
Der Hamilton-Operator des untersuchten $\mathrm{Mn}^{++}$. Spinsystems ist

$$
\mathfrak{S}_{\mathfrak{2}}(t)=\widetilde{S}_{0}+\widetilde{S}_{1}(t)
$$

mit dem zeitunabhängigen Teil

$$
\widetilde{\mathfrak{S}}_{0}=g_{I} \beta \boldsymbol{H} \cdot \mathbf{I}+A \boldsymbol{K} \cdot \mathbf{I}
$$

( $g_{I}=g$-Faktor, $I=$ Elektronenspin, $K=$ Kernspin von $\mathrm{Mn}^{++}, \boldsymbol{H}=$ magnetische Feldstärke, $A=$ Konstante der Hyperfeinwechselwirkung, $\beta=$ Bohrsches Magneton) und dem zeitabhängigen Teil

$$
\mathfrak{S}_{1}(t)=\mathscr{S}_{\mathrm{G}}(t)+\mathscr{S}_{\mathrm{D}}(t) \text {. }
$$

Der Anteil $\mathfrak{S}_{G}(t)$ charakterisiert die Spin-GitterWechselwirkung der $\mathrm{Mn}^{++}$.Ionen; sein Einfluß auf die $\mathrm{Mn}^{++}$-Linienbreite kann nicht explizit berechnet werden, er wird über die diamagnetischen Salzlösungen dem Experiment entnommen.

$\breve{S}_{\mathrm{D}}(t)$ beschreibt die magnetische Dipolwechselwirkung ${ }^{10}$ zwischen $\mathrm{Mn}^{++}$und $\mathrm{Co}^{++}, \mathrm{Ni}^{++}$bzw. $\mathrm{Ce}^{3+}$. Eine zusätzliche Austauschwechselwirkung zwischen den Ionen kann vernachlässigt werden, da keine Abnahme der Aufspaltung der Hyperfeinstruktur ${ }^{12}$ des $\mathrm{Mn}^{++}$-Spektrums beobachtet wurde.

Unter der Annahme, daß die Elektronenspinrelaxation exponentiell verläuft, kann die transversale Relaxationszeit $T_{2}$ nach Redfield ${ }^{13}$ berechnet werden.

Sofern zwischen Spin-Gitter- und Spin-Spin-Wechselwirkung keine Korrelation besteht, tragen $\mathscr{F}_{\mathfrak{G}}(t)$ und $\mathscr{S}_{\mathrm{D}}(t)$ additiv zu $1 / T_{2}$ bei :

$$
\frac{1}{T_{2}}=\frac{1}{T_{2}{ }^{(\mathrm{G})}}+\frac{1}{T_{2}^{(\mathrm{D})}} .
$$

Für den Beitrag der magnetischen Dipolwechselwirkung zu $1 / T_{2}$ errechnet man:
$\frac{1}{T_{2}{ }^{(\mathrm{D})}}=\frac{\beta^{4}}{\hbar^{2}} g_{I^{2}} g_{s}^{2} S(S+1)\left[\frac{1}{6} J_{0}\left(\tau_{1} ; 0\right)+\frac{1}{24} J_{0}\left(\tau_{2} ; \omega_{I}-\omega_{s}\right)\right.$ $\left.+\frac{3}{4} J_{1}\left(\tau_{1} ; \omega_{I}\right)+\frac{3}{2} J_{1}\left(\tau_{2} ; \omega_{s}\right)+\frac{3}{8} J_{2}\left(\tau_{2} ; \omega_{I}+\omega_{s}\right)\right]$.

$g_{s}=g$-Faktor, $S=$ effektiver Spin der Ionen mit kurzer Relaxationszeit $\left(\mathrm{Co}^{++}, \mathrm{Ni}^{++}, \mathrm{Ce}^{3+}\right), \omega_{s}=g_{s} \beta H / \hbar$, $\omega_{l}=\left(g_{l} \beta H+A m_{\mathrm{K}}\right) / \hbar, \tau_{1}$ bzw. $\tau_{2}=$ longitudinale bzw. transversale Relaxationszeit der Ionensorte mit Spin $S$.

Die Intensitätsfunktionen $J_{q}$ sind für isotrope Relaxation $\left(\tau_{1}=\tau_{2} \equiv \tau_{s}\right)$ bei PFEIFER ${ }^{11}$ angegeben.

10 A. Abragam, The Principles of Nuclear Magnetism, Oxford 1961.

11 H. Pfeifer, Ann. Phys. Leipzig 8, 1 [1961].

12 D. Kivelson, J. Chem. Phys. 27, 1087 [1957].
Aus dem Pfeiferschen Ausdruck für $J_{1}$ erhält man mit den Vereinfachungen $\omega \tau_{s} \ll 1$ und $\tau_{s} / \tau_{d} \ll 1$ (Definition von $\tau_{d}$ in Ref. ${ }^{11}$ ) :

$$
J_{1}\left(\tau_{s} ; \omega\right)=\frac{16 \pi C_{s} \tau_{s}}{45 a^{3}}
$$

( $a=$ minimal möglicher Abstand eines Ionenpaares $I-S)$. Mit $J_{1}$ und den Beziehungen ${ }^{11} J_{2}=4 J_{1}$ bzw.

13 A. G. Redfield, IBM J. Res. Develop. 1. 19 [1957]. - A. G. Redfield, in Adcanves in Magnetic Resonance, Vol. 1, Pergamon Press, New York 1964, p. 1. 
$J_{0}=6 J_{1}$ wird

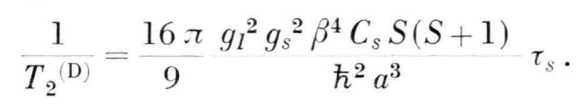

Im Falle $\tau_{1} \neq \tau_{2}$ ist $\tau_{s}$ der Mittelwert

$$
\tau_{s} \equiv \frac{1}{20}\left(7 \tau_{1}+13 \tau_{2}\right) \text {. }
$$

Da nach obiger Annahme das Spinsystem exponentiell relaxieren soll, zeigt die Hfs-Linie Lorentz-Form - was mit der experimentell beobachteten Linie in guter Näherung übereinstimmt - und zwischen Linienbreite $\Delta H$ und Relaxationszeit $T_{2}$ besteht der Zusammenhang

$$
\Delta H=\frac{2 \hbar}{\sqrt{3} g_{I} \beta T_{2}} .
$$

Der Beitrag $\triangle H_{\mathrm{D}}$ der Dipolwechselwirkung zur Gesamtlinienbreite $\Delta H$ ist daher

$$
\begin{aligned}
& \Delta H_{\mathrm{D}}=\frac{2 \hbar}{\sqrt{ } 3 g_{I} \beta T_{2}{ }^{(\mathrm{D})}} \\
& =\frac{32 \pi}{9 \sqrt{ } 3} \frac{g_{l} g_{s}{ }^{2} \beta^{3} C_{s} S(S+1)}{\hbar a^{3}} \tau_{s} .
\end{aligned}
$$

\section{Ergebnisse und Diskussion}

Aus dem Meßergebnis $\Delta H_{\mathrm{D}}=B \cdot C_{s}$ für $\mathrm{Ni}^{++}$kann über die errechnete Beziehung (5) zwischen $\Delta H_{\mathrm{D}}$ und $\tau_{s}$ eine mittlere Relaxationszeit $\tau_{s}$ für das $\mathrm{Ni}^{++}$. Spinsystem bestimmt werden:

$$
\tau_{s}=\frac{9 \sqrt{3}}{32 \pi} \frac{\hbar B a^{3}}{g_{I} g_{s}^{2} \beta^{3} S(S+1)}
$$

$a=5,2 \AA$ Für den kleinsten Abstand $a$ der Spins $I$ und $S$ wird der doppelte Komplexionsradius bekannter paramagnetischer Ionen ${ }^{\mathbf{1 4}}$ angenommen.

$g_{l}=2 \quad g$-Faktor von $\mathrm{Mn}^{++}$.

$g_{s}=2,25 \quad g$-Faktor kristalliner hydratisierter Ni-Salze ${ }^{1}$.

$S=1 \quad$ Elektronenspin von $\mathrm{Ni}^{++}$.

Diese Daten ergeben für $\mathrm{Ni}^{++}: \tau_{s}=5 \cdot 10^{-12} \mathrm{sec}$ $\left(27^{\circ} \mathrm{C}\right)$. Wegen der Unsicherheit von $B$ und $a$ kann $\tau_{s}$ um 30 bis $50 \%$ von diesem Wert abweichen.

Für die anderen Ionen kann im Rahmen der Meß-

14 R. Hausser u. F. Noack, Z. Phys. 182, 93 [1964]. genauigkeit $B^{\prime}<B / 4$ angenommen und damit eine obere Grenze für $\tau_{s}$ abgeschätzt werden:

$$
\begin{array}{lll}
\tau_{s}<2 \cdot 10^{-12} \mathrm{sec} & \text { für } & \mathrm{Ce}^{3+}, \\
\tau_{s}<3 \cdot 10^{-13} \mathrm{sec} & \text { für } & \mathrm{Co}^{++} .
\end{array}
$$

Diese Werte stimmen gut mit den Daten überein, die aus Untersuchungen der Resonanz und Relaxation von Protonen in Lösungen paramagnetischer Ionen bekannt sind:

Aus der Konzentrationsabhängigkeit der Protonenrelaxationszeiten entnehmen MORgan und NOLle ${ }^{2}$

$$
\tau_{s}=3 \cdot 10^{-12} \mathrm{sec} \text { für } \mathrm{Ni}^{++} .
$$

Aus der Frequenzabhängigkeit der Protonenrelaxationszeiten erhält NoACK ${ }^{7}$ bei $25^{\circ} \mathrm{C}$ :

$$
\tau_{s}<2 \cdot 10^{-12} \mathrm{sec} \text { für } \mathrm{Co}^{++} \text {und } \mathrm{Ni}^{++} \text {. }
$$

Luz und Меiвоом ${ }^{4}$ entnehmen der Protonenresonanz in Methanol-Wasser-Mischungen bei $-60^{\circ} \mathrm{C}$ die Werte

und

$$
\begin{aligned}
& \tau \approx 3 \cdot 10^{-12} \mathrm{sec} \text { für } \mathrm{Ni}^{++} \\
& \tau_{s} \approx 5 \cdot 10^{-13} \mathrm{sec} \text { für } \mathrm{Co}^{++} \text {. }
\end{aligned}
$$

Nach HeLd ${ }^{6}$ folgt aus der Frequenzunabhängigkeit der Protonenrelaxationszeiten für $\mathrm{Ce}^{3+}$ die Abschätzung: $\tau_{s}<8 \cdot 10^{-13} \mathrm{sec}$.

\section{Schlußbemerkung}

Die verschiedenen Methoden zur Bestimmung kurzer Relaxationszeiten paramagnetischer Ionen - einschließlich der hier angewandten - unterscheiden sich nicht wesentlich: Annahmen über ein Bewegungsmodell müssen gemacht und unbekannte atomistische Daten - wie z. B. der Komplexionenradius - müssen geschätzt werden. Exakte Werte sind offenbar erst dann zu erwarten, wenn es gelingt, Relaxationszeiten in der Größenordnung von $10^{-12}$ sec direkt zu messen.

Wir danken Herrn Prof. Dr. H. O. Kneser, der diese Arbeit ermöglicht und unterstützt hat. Die Arbeit wurde von der Deutschen Forschungsgemeinschaft maßgeblich gefördert. 